\title{
CLASSIFYING TIGHT WEYL-HEISENBERG FRAMES
}

\author{
Peter G. Casazza and Ole Christensen
}

\begin{abstract}
A Weyl-Heisenberg frame for $L^{2}(R)$ is a frame consisting of translates and modulates of a fixed function in $L^{2}(R)$, i.e. $\left(E_{m b} T_{n a} g\right)_{m, n \in Z}$, with $a, b>0$, and $g \in L^{2}(R)$. In this paper we will give necessary and sufficient conditions for this family to form a tight WH-frame. This allows us to write down explicitly all functions $g$ so that $\left(E_{m b} T_{n a} g\right)$ is an orthonormal basis for $L^{2}(R)$. These results give a simple direct classification of the alternate dual frames to Weyl-Heisenberg frames (a result due to Janssen).
\end{abstract}

\section{INTRODUCTION}

In 1946 D. Gabor [7] introduced a technique for signal processing which soon became a paradigm for the spectral analysis associated with time-frequency methods. His introduction of the short-time (windowed) Fourier transform led eventually to Wavelet theory. In 1952, Duffin and Schaeffer [5] introduced frame theory which put Gabor's technique into the framework of a larger model - that of a frame for a Hilbert space.

A sequence $\left(f_{i}\right)_{i \in I}$ of elements of a Hilbert space $H$ is called a frame for $H$ if there are constants $A, B>0$ so that

$$
A\|f\|^{2} \leq \sum_{i \in I}\left|<f, f_{i}>\right|^{2} \leq B\|f\|^{2}, \quad \text { for all } f \in H .
$$

The numbers $A, B$ are called the lower (resp. upper) frame bounds. The frame is a tight frame if $A=B$ and a normalized tight frame if $A=B=1$. If $\left(f_{i}\right)$ is a frame, the frame operator is defined by $S: H \rightarrow H, S f=\sum<f, f_{i}>f_{i}$. Then $S$ is bounded and invertible, and

$$
f=\sum_{i \in I}<S^{-1} f, f_{i}>f_{i}=\sum_{i \in I}<f, S^{-1 / 2} f_{i}>S^{-1 / 2} f_{i} .
$$

The first author was supported by NSF DMS 970618. The second author was supported by the Danish Research Council and would also like to thank the University of Missouri for their hospitality. 
One can check that $\left(f_{i}\right)_{i \in I}$ is a normalized tight frame if and only if $S=I$. One interpretation of (1.2) is that for an arbitrary frame $\left(f_{i}\right)$, a normalized tight frame equivalent to $\left(f_{i}\right)_{i \in I}$ is given by $\left(S^{-1 / 2} f_{i}\right)_{i \in I}$. Recall that Two frames $\left(f_{i}\right)$ and $\left(g_{i}\right)$ for a Hilbert space $H$ are equivalent if the operator $T: H \rightarrow H$ given by $T\left(f_{i}\right)=g_{i}$ is a well defined function which is an isomorphism of $H$ onto $H$.

If we replace $f$ in (1.1) by $f_{j}$, we see that

$$
\left\|f_{j}\right\|^{4}+\sum_{i \in I-\{j\}}\left|<f_{j}, f_{i}>\right|^{2} \leq B\left\|f_{j}\right\|^{2} .
$$

This yields immediately the following remark:

Remark 1.1. For all $j \in I,\left\|f_{j}\right\|^{2} \leq B$. If $\left\|f_{j}\right\|^{2}=B$ then $f_{j} \perp \operatorname{span}_{i \neq j} f_{i}$. In particular, if $\left(f_{i}\right)_{i \in I}$ is a normalized tight frame, then $\left\|f_{j}\right\| \leq 1$, and $\left\|f_{j}\right\|=1$ if and only if $f_{j} \perp \operatorname{span}_{i \neq j} f_{i}$.

The particular frames of interest to us will be the Weyl-Heisenberg frames. To define these frames, let $a, b \in R$ and define the operators of modulation $E_{b}$, and translation $T_{a}$ for functions $f \in L^{2}(R)$ by:

$$
E_{b} f(x)=e^{2 \pi i b x} f(x),
$$

and

$$
T_{a} f(x)=f(x-a) .
$$

Given $g \in L^{2}(R)$, and $a, b>0$, we say that $(g, a, b)$ generates a WH-frame for $L^{2}(R)$ if $\left(E_{m b} T_{n a} g\right)_{m, n \in Z}$ is a frame for $L^{2}(R)$. The function $g$ is referred to as the mother wavelet. The numbers $a, b$ are the frame parameters, with $a$ being the shift parameter and $b$ the modulation parameter.

Let $\mathbf{P F}$ denote the set of functions $g \in L^{2}(R)$ for which $\left(E_{m b} T_{n a} g\right)$ has a finite upper frame bound. It is easily seen that if $g \in \mathrm{PF}$, then $g$ is bounded. Theorem 2.1 of Casazza and Christensen [1] yields a sufficient condition for $g \in \mathrm{PF}$ :

Proposition (Casazza/Christensen [1]). If

$$
\sum_{k \in Z}\left|\sum_{n \in Z} g(x-n a) \overline{g(x-n a-k / b)}\right| \leq B \quad \text { a.e., }
$$

then $g \in P F$.

It can be shown that a variation of this produces a necessary condition for $g \in \mathrm{PF}$. That is, if $g \in \mathrm{PF}$ then,

$$
\sum_{k \in Z}\left|\sum_{n \in Z} g(x-n a) \overline{g(x-n a-k / b)}\right|^{2}<\infty .
$$


This condition is clearly not strong enough to classify PF. Also, condition (1.3) for $g \in \mathrm{PF}$ is not necessary [2]. This condition and its relationship to the Walnut representation of the frame operator are examined in detail by Casazza, Christensen, and Janssen [2].

In the above proposition and in the rest of the paper, we will be working with infinite sums of the form:

$$
\sum_{n \in Z} g(x-n a) \overline{g(x-n a-k / b)}
$$

So let us discuss the question of convergence here, and ignore it for the rest of the paper.

Proposition 1.2. If $g \in L^{2}(R)$ then the series

$$
\sum_{n \in Z} g(x-n a) \overline{g(x-n a-k / b)}
$$

converges absolutely a.e.

Proof. Since $g, T_{k / b} g \in L^{2}(R)$ we have that $g \overline{T_{k / b} g} \in L^{1}(R)$. Also,

$$
\left\|g \overline{T_{k / b} g}\right\|_{L^{1}}=\int_{R}\left|g(x) T_{k / b}(x)\right| d x=\int_{0}^{a} \sum_{n \in Z}|g(x-n a) g(x-n a-k / b)| d x<\infty .
$$

It follows that

$$
\sum_{n \in Z}|g(x-n a) \overline{g(x-n a-k / b)}|<\infty \text { a.e. }
$$

We need a result that appeared the first time in [9]:

WH-Frame Identity. If $g \in L^{2}(R)$ and $f \in L^{2}(R)$ is bounded and compactly supported, then

$$
\sum_{n \in Z} \sum_{m \in Z}\left|<f, E_{m b} T_{n a} g>\right|^{2}=F_{1}(f)+F_{2}(f)
$$

where

$$
\begin{gathered}
F_{1}(f)=b^{-1} \int_{R}|f(x)|^{2} \sum_{n}|g(x-n a)|^{2} d x \\
F_{2}(f)=b^{-1} \sum_{k \neq 0} \int_{R} \overline{f(x)} f(x-k / b) \sum_{n} g(x-n a) \overline{g(x-n a-k / b)} d x=
\end{gathered}
$$




$$
b^{-1} \sum_{k \geq 1} 2 R e \int_{R} \overline{f(x)} f(x-k / b) \sum_{n} g(x-n a) \overline{g(x-n a-k / b)} d x .
$$

To simplify the notation a little we introduce the following auxilliary functions:

$$
G(x)=\sum_{n \in Z}|g(x-n a)|^{2}
$$

and for all $k \in Z$,

$$
G_{k}(x)=\sum_{n \in Z} g(x-n a) \overline{g(x-n a-k / b)}
$$

It follows that $G_{0}=G$, and $G_{k}$ are periodic functions on $R$ of period a.

\section{Classifying Tight WH-Frames}

Now we will classify the tight WH-frames both abstractly in terms of the behavior of related families of vectors and concretely in terms of the behavior of the functions $G_{k}(x)$. In the next section we will write down explicitly the functions which satisfy the conditions of our theorem for some cases. Some of

We start with a basic fact which will simplify parts of the theorem and will be used in section 4 to classify the alternate dual frames for a WH-frame.

Proposition 2.1. Let $g, h \in L^{2}(R)$ and $a, b \in R$.

(1) $h \perp E_{m b} g$ for all $m \neq 0$ if and only if there is a constant $C$ so that

$$
\sum_{n \in Z} h(x-n / b) \overline{g(x-n / b)}=C, \quad \text { a.e.. }
$$

(2) If $n \neq 0$, then $h \perp E_{m b} T_{n a} g$, for all $m \in Z$ if and only if,

$$
\sum_{k} h(x-k / b) \overline{g(x-k / b-n a)}=0, \quad \text { a.e.. }
$$

Proof. (2): We just calculate:

$$
\begin{aligned}
<h, E_{m b} T_{n a} g> & =\int_{R} h(x) \overline{E_{m b} g(x-n a)} d x=\int_{R} h(x) \overline{g(x-n a)} E_{-m b} d x= \\
& \int_{0}^{1 / b} \sum_{k \in Z} h(x-k / b) \overline{g(x-k / b-n a)} E_{-m b} d x .
\end{aligned}
$$

(2) follows immediately from here and (1) is just the case of $n=0$ in (2).

We are now ready to prove the classification theorem for tight WH-frames. The equivalence of (1) and (2) in this theorem was first done by Janssen [6], Section 1.3.2. Also, the equivalence of (1) and (3) could be derived from results of Janssen [11], Theorem 3.1. But our proof is is simple and follows directly from the definitions. 
Theorem 2.2. Let $g \in L^{2}(R)$ and $a, b \in R$. The following are equivalent:

(1) $\left(E_{m b} T_{n a} g\right)_{n, m \in Z}$ is a normalized tight Weyl-Heisenberg frame for $L^{2}(R)$.

(2) We have:

(a) $G(x)=\sum_{n \in Z}|g(x-n a)|^{2}=b$ a.e.,

(b) $G_{k}(x)=\sum_{n \in Z} g(x-n a) \overline{g(x-n a-k / b)}=0$ a.e. for all $k \neq 0$.

(3) We have, $g \perp E_{n / a} T_{m / b} g$, for all $(n, m) \neq(0,0)$, and $\|g\|^{2}=a b$.

(4) $\left(E_{n / a} T_{m / b} g\right)_{n, m \in Z}$ is an orthogonal sequence in $L^{2}(R)$ and $\|g\|^{2}=a b$.

(5) $\left(E_{m b} T_{n a} g\right)_{n, m \in Z}$ is a Weyl-Heisenberg frame for $L^{2}(R)$ with frame operator $S$ and $S g=g$.

Moreover, in case the conditions are satisfied, $\left(E_{m b} T_{n a} g\right)_{m, n \in Z}$ is an orthonormal basis for $L^{2}(R)$ if and only if $\|g\|=1$.

Proof. (1) $\Rightarrow(2)$ : Assume $\left(E_{m b} T_{n a} g\right)_{n, m \in Z}$ is a normalized tight frame for $L^{2}(R)$. For any function $f \in L^{2}(R)$ which is bounded and supported on an interval of length $<1 / b$ we have for all $x \in R$ and all $0 \neq k \in Z$ that $\overline{f(x)} f(x-k / b)=0$. That is, $F_{2}(f)=0$. Now, by the WH-frame Identity:

$$
\begin{aligned}
\|f\|^{2} & =\int_{R}|f(x)|^{2} d x=\sum_{n} \sum_{m}\left|<f, E_{m b} T_{n a} g>\right|^{2}=F_{1}(f)+F_{2}(f) \\
& =b^{-1} \int_{R}|f(x)|^{2} \sum_{n}|g(x-n a)|^{2} d x=b^{-1} \int_{R}|f(x)|^{2} G(x) d x .
\end{aligned}
$$

Since this equality holds for all bounded $f \in L^{2}(I)$, for any interval $I$ of length $<1 / b$, it follows easily that $G(x)=b$, a.e. Hence, for all $f \in L^{2}(R), F_{1}(f)=\|f\|^{2}$. But now, again by the WH-frame Identity, we have for all bounded, compactly supported $f \in L^{2}(R)$,

$$
\|f\|^{2}=F_{1}(f)+F_{2}(f)=\|f\|^{2}+F_{2}(f) .
$$

That is, $F_{2}(f)=0$, for all bounded compactly supported $f \in L^{2}(R)$. Now fix $k_{0} \geq 1$ and let $I$ be any interval in $R$ of length $\leq 1 / b$. Define a function $f \in L^{2}(R)$ by:

$$
f(x)=e^{i \arg G_{k_{0}}(x)}, \text { for all } x \in I,
$$

and $f\left(x-k_{0} / b\right)=1$ for all $x \in I$ and $f(x)=0$, otherwise. Then, by the WH-frame Identity,

$$
0=F_{2}(f)=b^{-1} \sum_{k \geq 1} 2 \operatorname{Re} \int_{R} \overline{f(x)} f(x-k / b) \sum_{n} g(x-n a) \overline{g(x-n a-k / b)} d x=
$$




$$
b^{-1} 2 \operatorname{Re} \int_{R} \overline{f(x)} f\left(x-k_{0} / b\right) G_{k_{0}}(x) d x=b^{-1} 2 \int_{I}\left|G_{k_{0}}(x)\right| d x
$$

It follows that $G_{k_{0}}(x)=0$, a.e. on $I$. Since $k_{0}$ and $I$ were arbitrary, we have $(2 b)$.

$(2) \Rightarrow(1)$ : By assumption $(2 b)$ and the WH-frame Identity, we have $F_{2}(f)=0$ for all bounded, compactly supported $f \in L^{2}(R)$. Hence, applying assumption $(2 a)$ and the WH-frame Identity:

$$
\begin{gathered}
\sum_{n} \sum_{m}\left|<f, E_{m b} T_{n a} g>\right|^{2}=F_{1}(f)=b^{-1} \int_{R}|f(x)|^{2} \sum_{n}|g(x-n a)|^{2} d x= \\
\int_{R}|f(x)|^{2} d x=\|f\|^{2} .
\end{gathered}
$$

Since this equality holds on a dense subset of $L^{2}(R)$, it holds for all $f \in L^{2}(R)$. So $\left(E_{m b} T_{n a} g\right)_{n, m \in Z}$ is a normalized tight frame for $L^{2}(R)$.

$(2) \Leftrightarrow(3)$ : By (2) of Proposition 2.1, (2b) is equivalent to $g \perp E_{n / a} T_{m / b} g$, for all $m \neq 0$. Also, we have,

$$
\|g\|^{2}=\int_{R}|g(x)|^{2} d x=\int_{0}^{a} \sum_{n \in Z}|g(x-n a)|^{2} d x=\int_{0}^{a} G(x) d x .
$$

So the rest of the equivalence follows from (1) of Proposition 2.1.

$(3) \Leftrightarrow(4)$ : This is immediate from the observation that for all $m, n, \ell, k \in Z$ we have:

$$
<E_{n / a} T_{m / b} g, E_{k / a} T_{\ell / b} g>=e^{2 \pi i \frac{n-k}{a} \frac{m}{b}}<g, E_{\frac{k-n}{a}} T_{\frac{\ell-m}{b}} g>.
$$

$(1) \Leftrightarrow(5)$ : Since $S$ commutes with $E_{m b} T_{n a}$, we have that $S=I$ if and only if $S g=g$.

For the moreover part of the theorem, we just observe that for all $m, n \in Z$, $\|g\|=\left\|E_{m b} T_{n a} g\right\|$. Hence, if $\|g\|=1$, then our tight frame consists of norm 1 elements which now form an orthonormal basis by Remark 1.1.

With the characterization in Theorem 2.2, we can now recover easily and directly from the definition the basic properties of WH-frames which formerly required some more work. Janssen [10,11] first derived (1) in Corollary 2.3 below in an easier fashion. For any WH-frame $\left(E_{m b} T_{n a} g\right)_{m, n \in Z}$ with frame operator $S$, a direct computation shows that

$$
S\left(E_{m b} T_{n a} g\right)=E_{m b} T_{n a} S g, \text { for all } m, n \in Z \text {. }
$$

It follows that $S^{-1 / 2}$ also commutes with $E_{m b} T_{n a}$ and so $\left(E_{m b} T_{n a} S^{-1 / 2} g\right)_{m, n \in Z}$ is a normalized tight WH-frame which is equivalent to $\left(E_{m b} T_{n a} g\right)_{m, n \in Z}$ and hence 
must satisfy the conditions of Theorem 2.2. In particular, for all $(m, n) \neq(0,0)$ we have:

$$
<S^{-1} g, E_{n / a} T_{m / b} g>=<S^{-1 / 2} g, E_{n / a} T_{m / b} S^{-1 / 2} g>=0 .
$$

Corollary 2.3. Let $\left(E_{m b} T_{n a} g\right)_{m, n \in Z}$ be a WH-frame for $L^{2}(R)$.

(1) $S^{-1} g \perp E_{n / a} T_{m / b} g$, for all $(m, n) \neq(0,0)$ and

$$
<S^{-1} g, g>=<S^{-1 / 2} g, S^{-1 / 2} g>=\left\|S^{-1 / 2} g\right\|^{2}=a b \leq 1
$$

(2) If $a b<1$ then the WH-frame is not a Riesz basis.

(3) If $a b=1$ then the WH-frame is a Riesz basis for $L^{2}(R)$.

Proof. (1): All of this was observed before we stated the theorem except the last inequality $a b \leq 1$ which follows immediately from Remark 1.1.

(2): If our WH-frame is exact then $\left(E_{m b} T_{n a} S^{-1 / 2} g\right)$ is an orthonormal basis. Hence,

$$
1=\left\|S^{-1 / 2} g\right\|^{2}=a b .
$$

(3): If $a b=1$ then $\left(E_{m b} T_{n a} S^{-1 / 2} g\right)$ is a tight WH-frame and hence $\left(E_{n / a} T_{m / b} S^{-1 / 2} g\right)$ is an orthogonal sequence by Theorem $2.2(4)$. But, $a b=1$ implies that $n / a=n b$ and $m / b=n a$, so $\left(E_{n b} T_{m a} S^{-1 / 2} g\right)$ is an orthogonal basis for $L^{2}(R)$. Since $S^{-1 / 2}$ is an isomorphism, it follows that $\left(E_{m b} T_{n a} g\right)$ is a Riesz basis for $L^{2}(R)$.

Finally, we point out what appears to be a surprising consequence of Theorem 2.2 which seems to indicate the existence of a relationship between the values of a function and its Fourier Transform.

Corollary 2.4. If $g \in L^{2}(R)$ and $a b \leq 1$, the following are equivalent:

(1) The function $g$ satisfies:

$$
\sum_{n} g(x-n a) \overline{g(x-n a-k / b)}=0 \quad \text { a.e. for all } k \neq 0 \text {. }
$$

and

$$
\sum_{n}|g(x-n a)|^{2}=b \quad \text { a.e. }
$$

(2) The Fourier transform of the function $g$ satisfies:

$$
\sum_{n} \hat{g}(x-n b) \overline{\hat{g}(x-n b-k / a)}=0 \quad \text { a.e. for all } k \neq 0 \text {. }
$$


and

$$
\sum_{n}|\hat{g}(x-n b)|^{2}=a \quad \text { a.e. }
$$

Proof. Since the Fourier transform of $E_{m b} T_{n a} g$ is $E_{m a} T_{n b} \hat{g}$, Corollary 2.4 comes from Theorem 2.2 applied to the families $\left(E_{m b} T_{n a} g\right)$ and $\left(E_{m a} T_{n b} \hat{g}\right)$ which are normalized tight WH-frames together.

\section{The Functions $g$ Giving Tight Wh-Frames}

In this section we will give an explicit representation for the functions giving tight WH-frames given in Theorem $2.2(2)$ for the case $a=b=1$. If $f(x, y)$ is any function of two variables, we denote by $f_{y}(x)$ the function:

$$
f_{y}(x)=f(x, y)
$$

We start with a simple proposition which contains the basic notions which will be used in our characterization.

Proposition 3.1. The following are equivalent:

(1) The sequence $z=\left(c_{n}\right)_{n \in Z} \in \ell_{2}(Z)$ is orthogonal to all of its proper shifts and $\|z\|^{2}=b$.

(2) The unique function $h:[0,1] \rightarrow C$ with $\hat{h}(n)=c_{n}$, for all $n \in Z$ has $|h(x)|^{2}=b$ a.e.

(3) There is a measurable function $f:[0,1] \rightarrow R$ so that the function $h$ in (2) is of the form

$$
h(x)=\sqrt{b} e^{2 \pi i f(x)} .
$$

Proof. (1) $\Rightarrow(2)$ If $(\hat{h}(n))=\left(c_{n}\right)$ is orthogonal to all its shifts then $h \perp e^{2 \pi i m} h$, for all $m \neq 0$. That is, for all $m \neq 0$ we have

$$
0=<h, e^{2 \pi i m} h>=<|h|^{2}, e^{2 \pi i m}>
$$

Hence, $|h|^{2}=C$ a.e. Also, $\|h\|^{2}=\left\|\left(c_{n}\right)\right\|^{2}=b$.

$(2) \Rightarrow(3)$ : This is obvious.

$(3) \Rightarrow(1)$ : Given $h$ as in (3), for any $m \in Z$ we have:

$$
<h, E_{m} h>=\int_{0}^{1} h(x) \overline{h(x) E_{m}} d x=\int_{0}^{1}|h(x)|^{2} e^{-2 \pi i m x} d x=
$$




$$
\int_{0}^{1} b e^{-2 \pi i m x} d x=b \delta_{m} .
$$

So $h$ is orthogonal to $e^{2 \pi i m} h$ for all $m \neq 0$. Hence, $(\hat{h}(n))$ is orthogonal to all its proper shifts and $\|(\hat{h})(n)) \|^{2}=b$.

Now we are ready to give an explicit representation for the functions $g$ for which $\left(E_{m b} T_{n a} g\right)$ is an orthonormal basis for $L^{2}(R)$.

Theorem 3.2. Let $a b=1$ and $g \in L^{2}(R)$. The following are equivalent:

(1) $\left(E_{m b} T_{n a} g\right)$ is a orthonormal basis for $L^{2}(R)$.

(2) $\left(E_{m b} T_{n a} g\right)$ is a normalized tight WH-frame for $L^{2}(R)$.

(3) There is a measurable function $f:[0,1] \times[0, a) \rightarrow R$ and

$$
h(x, y)=\sqrt{b} e^{2 \pi i f(x, y)}
$$

so that

$$
g(y+n a)=\hat{h_{y}}(n), \text { for all } y \in[0, a)
$$

Proof. This is essentially immediate from our assumptions and Theorems 2.2 and 3.1. Since $a b=1$, we have $a=1 / b$ so Theorem 2.2 (2) becomes:

(a) $G(x)=\sum_{n \in Z}|g(x-n a)|^{2}=b$, a.e.,

(b) $G_{k}(x)=\sum_{n \in Z} g(x-n a) \overline{g(x-(n-k) a)}=0$, a.e. for all $k \neq 0$.

But condition (b) is equivalent to: $z_{y}=(g(y-n a))_{n \in Z}$ is orthogonal to all of its proper shifts and (a) is equivalent to $\left\|z_{y}\right\|^{2}=b$. By Theorem 3.1 these conditions are equivalent to: For each $y \in[0, a)$ there is a function $f_{y}:[0,1] \rightarrow R$ and $h_{y}:[0, a] \rightarrow C$ with

$$
h_{y}(x)=e^{2 \pi i f_{y}(x)}
$$

and

$$
\hat{h_{y}}(n)=g(y-n a)
$$

So defining $f(x, y):[0,1] \times[0, a) \rightarrow R$ and $h(x, y)$ by:

$$
f(x, y)=f_{y}(x), \quad h(x, y)=h_{y}(x)
$$

yields the theorem modulo the measurability conditions which are obvious. 


\section{Alternate Dual Frames}

If $\left(f_{i}\right)_{i \in I}$ is a frame for a Hilbert space $H$, a frame $\left(h_{i}\right)_{i \in I}$ for $H$ is called an alternate dual frame or a pseudo-dual for $\left(f_{i}\right)_{i \in I}$ if

$$
f=\sum_{i \in I}<f, h_{i}>f_{i}, \text { for all } f \in H
$$

We already know one sequence $\left(h_{i}\right)_{i \in I}$ satisfying (4.1). Namely, the sequence $\left(S^{-1} f_{i}\right)_{i \in I}$. We call $\left(S^{-1} f_{i}\right)_{i \in I}$ the canonical dual of $\left(f_{i}\right)$. If $\left(f_{i}\right)_{i \in I}$ is a normalized tight frame, then $S=I$, so the frame equals its canonical dual frame. The converse of this clearly holds also. But in general, there are many alternate dual frames for a given frame. For the basic properties of alternate dual frames we refer to $[7,9,12]$. Now we will use the techniques developed in section 3 to characterize the Weyl-Heisenberg alternate dual frames for a given Weyl-Heisenberg frame. We need a beautiful result of Wexler-Raz [13] (see also Janssen [10,11]):

Theorem(Wexler-Raz [13]). Let $g, h \in P F$. Then $\left(E_{m b} T_{n a} h\right)$ and $\left(E_{n / a} T_{m / b} g\right)$ are alternate dual frames if and only if both $h \perp E_{n / a} T_{m / b} g$, for all $(m, n) \neq(0,0)$, and $<h, g\rangle=a b$.

We proceed with the corresponding result to Theorem 2.2 for alternate dual frames. (1) $\Leftrightarrow(2)$ in the theorem below was first proved by Janssen [6], section 1.3.2.

Theorem 4.1. For $g, h \in \mathbf{P F}$ and $\left(E_{m b} T_{n a} g\right)$ a WH-frame for $L^{2}(R)$, the following are equivalent:

(1) $\left(E_{m b} T_{n a} h\right)_{m, n \in Z}$ is an alternate dual frame for $\left(E_{m b} T_{n a} g\right)_{m, n \in Z}$.

(2) We have:

(a) $\sum_{n \in Z} h(x-n a) \overline{g(x-n a-k / b)}=0$ a.e. for all $k \neq 0$.

(b) $\sum_{n \in Z} h(x-n a) \overline{g(x-n a)}=b$, a.e.

(3) $h=S^{-1} g+f$, where $f \in L^{2}(R)$ and $f \perp \operatorname{span}_{n, m \in Z} E_{n / a} T_{m / b} g$.

Proof. (1) $\Leftrightarrow(2)$ : This is Proposition 2.1 combined with the theorem of WexlerRaz.

(1) $\Rightarrow(3)$ : By the Wexler-Raz Theorem, $h \perp E_{n / a} T_{m / b} g$, for all $(m, n) \neq(0,0)$. By Corollary 2.3, we also have that $S^{-1} g \perp E_{n / a} T_{m / b} g$. Hence, $f=h-S^{-1} g \perp$ 
$E_{n / a} T_{m / b} g$. Again by the Wexler-Raz Theorem, $<h, g>=a b$ and applying Corollary 2.3 again,

$$
<h-S^{-1} g, g>=<h, g>+<S^{-1} g, g>=a b-a b=0
$$

It follows that $h=S^{-1} g+\left(h-S^{-1} g\right)=S^{-1} g+f$ and $f \perp E_{n / a} T_{m / b} g$, for all $n, m \in Z$.

$(3) \Rightarrow(1)$ : Fix $(m, n) \neq(0,0)$. We compute using Corollary 2.3:

$$
\begin{gathered}
<h, E_{n / a} T_{m / b} g>=<S^{-1} g+f, E_{n / a} T_{m / b} g>= \\
<S^{-1} g, E_{n / a} T_{m / b} g>+<f, E_{n / a} T_{m / b} g>=0+0=0 .
\end{gathered}
$$

Also, using Corollary 2.3,

$$
<h, g>=<S^{-1} g+f, g>=<S^{-1} g, g>+<f, g>=a b+0=a b \text {. }
$$

So this implication follows from the Wexler-Raz Theorem.

Note that $\left(E_{m b} T_{n a} g\right)_{m, n \in Z}$ is a normalized tight frame if and only if we can replace $S^{-1} g$ in Theorem 4.1 by the function $g$ and in this case Theorem 4.1 reduces to Theorem 2.2. Also in this case, $S=I$ so part (3) of the theorem becomes: $h=g+f$ where $f \perp \operatorname{span}_{n, m \in Z} E_{n / a} T_{m / b} g$.

ACKNOWLEDGEMENT. The authors express their deepest gatitude to A.J.E.M. Janssen for making extensive recommendations for improvements of this manuscript.

\section{REFERENCES}

1. P.G. Casazza and O. Christensen, Weyl-Heisenberg frames for subspaces of $L^{2}(R)$, (preprint).

2. P.G. Casazza, O. Christensen, and A.J.E.M. Janssen, Weyl-Heisenberg frames, translation invariant systems and the Walnut representation, (preprint).

3. I. Daubechies, The wavelet transform, time-frequency localization and signal analysis, IEEE Trans. Inf. Theory 36 (1990), 961-1005.

4. I. Daubechies, "Ten Lectures on Wavelets", CBMS-NSF regional conference series in applied mathematics, Philadelphia 61 (1992).

5. R.J. Duffin and A.C.Schaeffer, A class of non-harmonic Fourier series, Trans. Amer. Math. Soc. 72 (1952), 341-366.

6. H.G. Feichtinger, T. Strohmer Editors, Gabor analysis and Algorithms - Theory and Applications,, Birkhauser, Applied and Numerical Analysis Series (1998).

7. D. Gabor, Theory of communications, J. Inst. Elec. Eng. (London) 93 (1946), 429-457.

8. D. Han and D.R. Larson, Frames, Bases and Group Representations, (preprint).

9. C.E. Heil and D.F. Walnut, Continuous and discrete wavelet transforms, SIAM Review 31 (No. 4) (1989), 628-666. 
10. A.J.E.M. Janssen, Signal analytic proofs of two basic results on lattice expansions, Appl. Comp. Harm. Anal. 1 (4) (1994), 350-354.

11. A.J.E.M. Janssen, Duality and biorthogonality for Weyl-Heisenberg frames, J. Fourier Anal. and Appl. 1 (4) (1995), 403-437.

12. A. Ron and C. Shen, Weyl-Heisenberg frames and Riesz bases in $L^{2}(R)$, Duke Math Journal 89 No. 2 (1997), 237-282.

13. J. Wexler and S. Raz, Discrete Gabor expansions, signal processing 21 (1990), 207-220.

Department of Mathematics, The University of Missouri, Columbia, Missouri 65211 , USA and Mathematical Institute, Building 303, Technical University of Denmark, 2800 LYNGBY, DENMARK

E-mail address: pete@casazza.math.missouri.edu; olechr@mat.dtu.dk 